\title{
A CHARACTERIZATION OF THE CAUCHY TYPE
}

\author{
FRANK B. KNIGHT
}

\begin{abstract}
It is proved that a random variable $X$ is of Cauchy type if and only if $(a X+b)(c X+d)^{-1}$ has the same type as $X$ for every $a, b, c$, $d$ with $a d-b c \neq 0$.
\end{abstract}

The Cauchy density $f(y)=\pi^{-1}\left(1+y^{2}\right)^{-1},-\infty<y<\infty$, with distribution function $F(x)=\pi^{-1}\left(\operatorname{Tan}^{-1} x+\pi / 2\right)$, has come to play an increasingly important role both in advanced probability and in undergraduate courses. Much of this emphasis is certainly due to its position as a member of the symmetric stable family. Recalling that the "type" of a probability distribution $F$ is the equivalence class of $F$ under the relation $F_{1} \equiv F_{2}$ if either $F_{1}(x)$ $=F_{2}(a x+b), a>0$, or $F_{1}(x)=1-F_{2}(a x+b), a<0$, for constants $(a, b)$, we can define the symmetric stable family by the property that the distribution type is invariant under convolution.

In the present note, however, we will characterize the Cauchy type by the following property, for which convolution (i.e. sums of independent random variables) has no apparent relevance.

THEOREM. A random variable $X$ is of Cauchy type if and only if, whenever $a d-b c \neq 0,(a X+b)(c X+d)$ has the same type as $X$.

Remarks. A related result of E. J. Williams [5] asserts that $X$ is standard Cauchy if and only if, for some constant $b$ which is not the tangent of a rational multiple of $2 \pi$, both $X$ and $(1+b X) /(b-X)$ have the same distribution. The proof of [5] is a fairly transparent application of the representation $X=\tan \theta$, where $\theta$ is uniformly distributed on $(0, \pi)$ if $X$ is standard Cauchy. We have not found any way to use this representation for the present theorem, however, nor do we know of any physical or geometrical reasoning by which one can deduce the result. This seems to be a gap, since the theorem has an obvious geometric interpretation in terms of the group $y=(a x+b) /(c x+d)^{-1}, a d-b c \neq 0$, of projectivities of the line. It is equivalent to the assertion that the Cauchy measure type is the only finite measure type invariant under projection from one line onto another in the Euclidean plane, from any point not on either line. ${ }^{1}$

Proof. By straightforward algebra, if $c \neq 0,(a X+b) /(c X+d)^{-1}=c_{1}$ $+c_{2}\left(X+c_{3}\right)^{-1}$, where $c_{3}=d / c$, and this has the same type as $\left(X+c_{3}\right)^{-1}$.

Received by the editors October 8, 1974 and, in revised form, January 20, 1975.

AMS (MOS) subject classifications (1970). Primary 60E05; Secondary 28-01.

Key words and phrases. Cauchy distribution, type, projectivity, invariance, characterization.

${ }^{1}$ ADDED IN PROOF. This gap is filled in [2] using more advanced methods. 
Setting $c_{3}=c$, it thus suffices to consider only invariance of type for $(X+c)^{-1}$. If $X$ is standard Cauchy, it is just an exercise to show that the density of $(X+c)^{-1}$ is $a f(a y+b)$ where $a=1+c^{2}$ and $b=-c$, a fact used previously in [3]. This proves sufficiency, since any $X^{\prime}$ of Cauchy type is distributed like $c_{1} X+c_{2}$, hence $\left(X^{\prime}+c\right)^{-1}$ has the same type as $\left(X+\left(c_{2}+c\right) / c_{1}\right)^{-1}$.

Our proof of the necessity is considerably more involved. We begin by noting that the distribution $F_{X}(x)$ of $X$ must be continuous, for at a discontinuity $-c,(X+c)^{-1}$ would not even have a well-defined distribution.

LEMMA 1. $F_{X}=F$ has a strictly positive and continuous density $f(y)$.

Proof. For $x>0$ we have

$$
\begin{aligned}
P\left\{(X+c)^{-1} \leqq x\right\} \\
\quad=P\left\{X+c \leqq x^{-1} \text { and } X+c>0\right\}+P\left\{x^{-1} \leqq X+c \text { and } X+c<0\right\} \\
\quad=P\left\{-c<X<-c+x^{-1}\right\}+P\{X<-c\} .
\end{aligned}
$$

Assuming that $F_{(X+c)^{-1}}(x)=F(a(c) x+b(c))$, for $a(c)>0$, we thus have

$$
F\left(-c+x^{-1}\right)-F(-c)=1-F(a(c) x+b(c)),
$$

while for $a(c)<0$ the right side is simply $F(a(c) x+b(c))$. The left side is continuous in $(c, x)$ for $x \neq 0$, while $F(x)$ must have (uncountably many) points of two-sided increase. For each $c$ we can thus set $a(c) x_{1}+b(c)$ and $a(c) x_{2}+b(c)$ equal to two such points, and it follows that $a(c)$ (which has no zeros) and $b(c)$ are each continuous in $c$. If we now divide (1) by $x^{-1}$ and take the limit inferior and the limit superior as $x \rightarrow \infty$, the right side becomes

$$
\lim \inf _{x \rightarrow \infty} \frac{x(1-F(x))}{a(c)} \text { or } \lim \sup _{x \rightarrow \infty} \frac{x(1-F(x))}{a(c)}
$$

respectively, hence each is either $\equiv 0, \equiv \infty$, or $k a(c)^{-1}, k>0$, for all $c$. An analogous result holds for $a(c)<0$, and since the left side of (1) becomes $d^{+} F(-c) / d x^{+}$, except perhaps on a Lebesgue null set, the two cases of lim sup and lim inf merge. The alternatives 0 and $\infty$ are excluded by the nature of $F$, the former because $d^{+} F / d x^{+} \equiv 0$ implies that $F$ is a constant [4, p. 98]. Hence

$$
F(x)=\int_{-\infty}^{x} \frac{d^{+}}{d y^{+}} F(y) d y=\int_{-\infty}^{x} k|a(y)|^{-1} d y
$$

and the lemma is proved.

Differentiating in (1) we now obtain equivalently

$$
x^{-2} f\left(x^{-1}-c\right)=|a(c)| f(a(c) x+b(c)) .
$$

The proof of the following two lemmas is postponed to follow the main argument.

LEMMA 2. $a(c)>0$. 
Lemma 3. $f(y)$ has 3 continuous derivatives.

Using these lemmas, we shall conclude the argument. Setting $h(y)$ $=f^{-1}(y),(2)$ is equivalent to

$$
x^{2} h\left(x^{-1}-c\right)=(a(c))^{-1} h(a(c) x+b(c)) .
$$

Next, if we set $x^{-1}-c=a(c) x+b(c)$, we have a quadratic equation in $x$ with discriminant $(b(c)+c)^{2}+4 a(c)>0$. Hence, real solutions exist, and at such a solution $x_{0}$ we have $a(c) x_{0}^{2}=1-(b(c)+c) x_{0}$, while at the same time, by cancelling $h$ in (3), $x_{0}^{2}=a(c)^{-1}$. Thus $b(c)=-c$. Next, we differentiate twice in (3), obtaining

$$
2 x h\left(x^{-1}-c\right)-h^{\prime}\left(x^{-1}-c\right)=h^{\prime}(a(c) x-c),
$$

and

(5) $2 h\left(x^{-1}-c\right)-2 x^{-1} h^{\prime}\left(x^{-1}-c\right)+x^{-2} h^{\prime \prime}\left(x^{-1}-c\right)=a(c) h^{\prime \prime}(a(c) x-c)$.

Therefore, irrespective of the sign of $x$, we have

$$
\lim _{x \rightarrow \pm \infty} a(c) h^{\prime \prime}(x)=2 h(-c),
$$

and it follows that $h^{\prime \prime}$ must have an interior extremum, denoted by $x^{\prime}$. Now if we introduce $w=x^{-1}$ in (5) and differentiate with respect to $w$, cancellation occurs and we are left with

$$
w^{2} h^{\prime \prime \prime}(w-c)=-a^{2}(c) w^{-2} h^{\prime \prime \prime}\left(a(c) w^{-1}-c\right) .
$$

The right side vanishes whenever $a(c) w^{-1}-c=x^{\prime}$, or $w=a(c)\left(c+x^{\prime}\right)^{-1}$. As $c \rightarrow-x^{\prime} \pm$, we obtain that $h^{\prime \prime \prime}(x)=0$ for all $|x|$ sufficiently large. But writing (7) in the form

$$
h^{\prime \prime \prime}\left(x^{-1}-c\right)=-a^{2}(c) x^{4} h^{\prime \prime \prime}(a(c) x-c)
$$

and letting $x \rightarrow \infty$, it follows that $h^{\prime \prime \prime}(-c) \equiv 0$. Hence, $h(x)=f^{-1}(x)$ is a polynomial of degree 2 , and by routine completion of the square it must have the form $a^{-1}\left((a x+b)^{2}+1\right), a>0$, in order for $f$ to be a probability density. This completes the proof.

Returning to Lemma 2, our proof is by a (somewhat tedious) reductio ad absurdum. By (2) we have, if $a(c)<0$,

$$
\lim _{x \rightarrow \pm \infty} f\left(x^{-1}-c\right)=\lim _{x \rightarrow \pm \infty}-a(c)^{-1} x^{2} f(x)=-k a(c)^{-1}
$$

where $k>0$ is a constant. Letting $x^{-1}-c=z$, and noting that $f(-c)$ $=-k a(c)^{-1}$, we obtain by (2) and (9)

$$
\begin{aligned}
f(z) & =-a(c)(z+c)^{-2} f\left(a(c)(z+c)^{-1}+b(c)\right) \\
& =\lim _{c \rightarrow \pm \infty} f\left(a(c)(z+c)^{-1}+b(c)\right),
\end{aligned}
$$

uniformly in finite intervals of $z$. The argument on the right equals 
$(a(c)+c b(c))(c+z)^{-1}+b(c)(c+z)^{-1} z$, and as $c \rightarrow \pm \infty$, this must converge to a linear function $\alpha+\beta z$. Since $f(z)=f(\alpha+\beta z)$ and $\lim _{z \rightarrow \pm \infty} f(z)=0$, we can obtain by iteration that $|\beta|=1$ and $\alpha=0$. But since $a(c)<0$, and $\lim _{c \rightarrow \infty} c^{-1}(a(c)+c b(c))=0$, we must have

$$
\beta=+1=\lim _{c \rightarrow \pm \infty} b(c) c^{-1}=\lim _{c \rightarrow \pm \infty}-\frac{a(c)}{c^{2}} .
$$

Next, we make the simultaneous substitutions $(c+z) a(c)^{-1}$ for $x$ and $b(c)$ for $c$ in (2) to get

$$
\begin{aligned}
a(c)^{2}(c & +z)^{-2} f\left(a(c)(c+z)^{-1}-b(c)\right) \\
& =-a(b(c)) f\left(\frac{a(b(c))}{a(c)}(c+z)+b(b(c))\right) .
\end{aligned}
$$

Letting $z \rightarrow-c$ and recalling (9) and (2) we have

$$
k=-a(b(c)) f(b(b(c)))=k f^{-1}(-b(c)) f(b(b(c))) .
$$

Since the range of $b(c)$ is $(-\infty,+\infty)$, this yields for all $x$,

$$
f(b(x))=f(-x) .
$$

Finally, we consider the simultaneous equations $-\left(x^{-1}-c\right)=w$ and $a(c) x$ $+b(c)=b(w)$, for fixed $c$. Whenever there is a solution $(x, w)$ we can cancel in (2), using (14), to obtain $x^{-2}=-a(c)$. These equations become

$$
x=(c-w)^{-1} \text { and } x=(b(w)-b(c)) a(c)^{-1},
$$

where the latter is continuous in $w$ with limits $+\infty$ and $-\infty$ as $w$ $\rightarrow-\infty$ and $w \rightarrow+\infty$. It follows that for each $c$ there are two simultaneous solutions, one at $w_{1}(c)<c$, and one at $w_{2}(c)>c$, with corresponding values $x= \pm \sqrt{-a(c)}$. By algebra we have

$$
w_{2}(c)-w_{1}(c)=2(-a(c))^{-\frac{1}{2}}
$$

and

$$
b\left(w_{2}(c)\right)-b\left(w_{1}(c)\right)=2(-a(c))^{\frac{3}{2}}=a(c)^{2}\left(w_{2}(c)-w_{1}(c)\right) .
$$

Since $\lim _{c \rightarrow \infty} c(-a(c))^{-\frac{1}{2}}=1$, we can choose inductively $0<c_{1}<c_{2}<\cdots$ such that $w_{2}\left(c_{n}\right)=w_{1}\left(c_{n+1}\right)$ for each $n$, and $\lim _{n \rightarrow \infty} c_{n}=\lim _{n \rightarrow \infty} w_{2}\left(c_{n}\right)$ $=\infty$. Thus, for each $n$ we obtain

$$
\begin{aligned}
b\left(w_{2}\left(c_{n}\right)\right)-b\left(w_{1}\left(c_{2}\right)\right) & =\sum_{k=2}^{n} b\left(w_{2}\left(c_{k}\right)\right)-b\left(w_{1}\left(c_{k}\right)\right) \\
& =\sum_{k=2}^{n} a\left(c_{k}\right)^{2}\left(w_{2}\left(c_{k}\right)-w_{2}\left(c_{k-1}\right)\right),
\end{aligned}
$$

where $\lim _{k \rightarrow \infty} a\left(c_{k}\right)^{2} c_{k}^{-4}=1$. But this implies that $\lim _{n \rightarrow \infty} w_{2}\left(c_{n}\right) b\left(w_{2}\left(c_{n}\right)\right)^{-1}$ $=0$, contrary to (11), and completes the proof of Lemma 2 . 
Turning to the proof of Lemma 3, we note that, up to terms of smaller order of magnitude, the differential quotients of (3) yield

$$
\begin{aligned}
& x^{2}(\Delta x)^{-1}(\left.\left(x^{-1}-c-x^{-2} \Delta x\right)-h\left(x^{-1}-c\right)\right)+2 x h\left(x^{-1}-c\right) \\
&=(a(c) \Delta x)^{-1}(h(a(c) x-c+a(c) \Delta x)-h(a(c) x-c)) .
\end{aligned}
$$

Setting $y=x^{-1}-c=a(c) x-c$, we have as before the solution $x=a(c)^{-\frac{1}{2}}$. Thus $x^{-2} \Delta x=a(c) \Delta x=\Delta y$, and we can combine the first term on the left of (15) with the right side to obtain

$$
\lim _{\Delta y \rightarrow 0}(\Delta y)^{-1}(h(y+\Delta y)-h(y-\Delta y))=2 x h(y),
$$

where $y=a(c)^{\frac{1}{2}}-c$ and $x=a(c)^{-\frac{1}{2}}$ are continuous in $c$. This implies easily that

$$
h^{\prime}(y)=a(c)^{-\frac{1}{2}} h(y)
$$

for all $y$ of this form: in particular, for all $y$ sufficiently large (as $c \rightarrow-\infty$ ). But by (9) (with $a(c)>0$ in the present case) we have $h(-c)=k a^{-1}(c)$, whence $a^{\prime}(c)$ exists for all $c$ sufficiently large negative. Then we can differentiate (17) again repeatedly and obtain the existence of the derivatives of $h$ for large $y$. But it is clear from (3) that differentiability of $h(a(c) x+b(c))$ for large $x$ implies differentiability at $-c$ for arbitrary $c$. This completes the proof.

As was noted above, this theorem has an interpretation in terms of measures instead of probabilities. In fact, using the notation $y^{(-1)}(d x)=\{x: y(x) \in d x\}$ for any set $d x$, we have as a final observation the

Corollary. Let $\mu(d x)$ be a (possibly infinite) Borel measure which is finite on compact sets and such that, for every $y=(a x+b) /(c x+d)(a d-b c \neq 0)$ there is some $z=\alpha x+\beta$ for which $\mu\left(y^{(-1)}(d x)\right)=\mu\left(z^{(-1)}(d x)\right)$. Then $\mu(d x)$ is necessarily finite, and, hence, of Cauchy type. If $\mu$ is not assumed finite on compact sets, then either $\mu$ is finite or $\mu\left(x_{1}, x_{2}\right)=\infty$ for all $x_{1}<x_{2}$.

Proof. Choose $y(x)=1 / x-c$, so that $y^{(-1)}(x)=-1 /(x+c)$. Then writing $\mu(a, b)=\mu(b, a)$ if $a>b$, we have for suitable constants $\hat{\alpha}(c), \hat{\beta}(c)$ and $x_{1}$ $<-c<x_{2}$, the identity

$$
\mu\left(\hat{\alpha}(c) x_{1}+\hat{\beta}(c), \hat{\alpha}(c) x_{2}+\hat{\beta}(c)\right)=\mu\left(-\infty, 1 /\left(x_{1}+c\right)\right)+\mu\left(1 /\left(x_{2}+c\right), \infty\right) .
$$

Thus $\mu$ is finite at $\pm \infty$ and the first assertion follows.

For the second, we consider on the extended real line $\bar{R}=\{-\infty \leqq x \leqq \infty\}$ the set $S=\{x: \mu(N(x))=\infty$ for every open neighborhood $N(x)$ of $x\}$. Clearly, $S$ is closed in $\bar{R}$. If $S$ is not dense in $\bar{R}$, let $N \subset \bar{R}-S$ be open. Then for $c \in N$ and $y^{(-1)}(x)=-1 /(x+c)$ the measure $\mu\left(y^{(-1)}(d x)\right)$ is bounded at $\pm \infty$. Since $z^{(-1)}$ preserves boundedness, this implies that $\mu(d x)$ is itself bounded at $\pm \infty$, and thus $S$ is a bounded subset of $R$. But if $-c$ $\in S$ then $y^{(-1)}(x)$ takes $N(-c)$ into a neighborhood of $\{ \pm \infty\}$ while $z^{-1}(x)$ leaves $S$ bounded. This is a contradiction unless $S$ is void, completing the proof. 


\section{REFERENCES}

1. William Feller, An introduction to probability theory and its applications, Vol. II, Wiley, New York, 1966. MR 35 \# 1048.

2. F. B. Knight and P. A. Meyer, Une charactérisation de la loi de Cauchy, Z. Wahrscheinlichkeitstheorie und Verw. Gebiete (to appear).

3. E. J. G. Pitman and E. J. Williams, Cauchy-distributed functions of Cauchy variates, Ann. Math. Statist. 38 (1967), 916-918. MR 35 \#1060.

4. H. L. Royden, Real analysis, 2nd. ed., Macmillan, New York, 1968.

5. E. J. Williams, Cauchy-distributed functions and a characterization of the Cauchy distribution, Ann. Math. Statist. 40 (1969), 1083-1085. MR 39 \#4978.

Department of Mathematics, University of Illinois at Urbana-Champaign, Urbana, ILLINOIS 61801 